\title{
Distribución potencial del Pinus martinezii: un modelo espacial basado en conocimiento ecológico y análisis multicriterio
}

\section{Potential distribution of Pinus martinezii: an spatial model based in ecological knowledge and muticriteria analysis}

\author{
Óscar Leal-Nares ${ }^{1}$, Manuel E. Mendoza ${ }^{2 凶}$, Diego Pérez-Salicrup³, Davide Geneletti ${ }^{4,7}$, Erna López-Granados ${ }^{5}$ \\ y Eleazar Carranza ${ }^{6}$ \\ ${ }^{1}$ Especies, Sociedad y Hábitat, A.C. Los Alpes 301-3, Col. Jardín, 64050 Monterrey, Nuevo León, México. \\ ${ }^{2}$ Centro de Investigaciones en Geografía Ambiental, Universidad Nacional Autónoma de México. Antigua Carretera a Pátzcuaro 8701, Col. Ex \\ Hacienda de San José de la Huerta, 58190 Morelia, Michoacán, México. \\ ${ }^{3}$ Centro de Investigaciones en Ecosistemas, Universidad Nacional Autónoma de México, Antigua Carretera a Pátzcuaro 8701, Col. Ex Hacienda de San \\ José de la Huerta, 58190 Morelia, Michoacán, México. \\ ${ }^{4}$ Department of Civil and Environmental Engineering, University of Trento. Via Mesiano 77, Trento 38100 Italia. \\ ${ }^{5}$ Departamento de Geología y Mineralogía, Instituto de Investigaciones Metalúrgicas, Universidad Michoacana de San Nicolás de Hidalgo. Edificio U, \\ Ciudad Universitaria, Morelia, Michoacán, México. \\ ${ }^{6}$ Instituto de Ecología, A.C. Bajio, Av. Lázaro Cárdenas 253, 61600 Pátzcuaro, Michoacán, México. \\ ${ }^{7}$ Sustainability Science Program, Harvard University, 79 JFK Street, Cambridge, Massachusetts 02138, USA. \\ \mmendoza@ciga.unam.mx
}

\begin{abstract}
Resumen. El modelado de la distribución potencial y actual de las especies se ha convertido en un área de investigación muy activa; generalmente se basa en el concepto de nicho ecológico y se apoya en el uso de programas de cómputo. El objetivo principal de esta investigación fue elaborar un modelo de distribución potencial de Pinus martinezii en la cuenca del lago de Cuitzeo, utilizando información ambiental y datos de presencia de la especie, lo que requirió identificar los factores ambientales que determinan la distribución de P. martinezii, y elaborar un perfil bioclimático de la especie. El modelo se apoyó en un análisis multicriterio dentro de un sistema de información geográfica. Los atributos se agruparon en 3 criterios: geopedológicos, morfométricos y climáticos. De acuerdo con el mapa que se obtuvo, en la cuenca hay 2 zonas principales de distribución potencial de $P$. martinezii y algunas regiones aisladas donde no se encontraron poblaciones. El modelo espacial constituye una herramienta importante para planificar labores de conservación y reforestación; asimismo, puede utilizarse para planificar exploraciones en busca de nuevas poblaciones de P. martinezii que no han sido registradas, o identificar sitios donde esta especie pueda reintroducirse.
\end{abstract}

Palabras clave: modelo espacial SIG, perfil bioclimático, nicho ecológico, reforestación, conservación, Cuitzeo, México.

\begin{abstract}
The modeling of potential and current distribution of species has become a very active research area. Generally, modeling is based on the concept of ecological niche, and is supported by the use of computer programs. The main objective of this project was to develop a potential distribution model of Pinus martinezii in the Cuitzeo Lake basin using data of environmental variables, and presence of the target species. To this purpose, the environmental factors that determine the distribution of $P$. martinezii were identified, and a bioclimatic profile of the species was made. The modeling was based on a spatial multicriteria analyisis. The attributes were grouped into 3 criteria: geopedologic, morphometric and climatic conditions. The resulting potential distribution map showed that there are 2 main areas of potential distribution of $P$. martinezii, and some isolated areas where populations have not been found. The spatial model is an important tool for planning conservation and reforestation efforts, as well as to plan additional field surveys and identifying sites where the specie can be reintroduced.
\end{abstract}

Key words: spatial modeling GIS, bioclimatic profile, ecological niche, reforestation, conservation, Cuitzeo, Mexico.

\section{Introducción}

En la investigación para la conservación biológica, la estimación de las áreas de distribución potencial y actual

Recibido: 12 septiembre2011; aceptado: 13 abril 2012 de las especies mediante el modelado del nicho ecológico se ha convertido en una actividad importante (Soberón y Nakamura, 2009). Dentro de una comunidad, cada especie tiene un lugar propio en el espacio y tiempo que puede representarse en un mapa. La distribución espacial de las especies no es aleatoria, sino que obedece al 
intervalo o capacidad de tolerancia que cada especie tiene a factores ambientales como altitud, posición topográfica, temperatura, humedad y precipitación que Chapman (1976) definió como amplitud ecológica. En el caso específico de las plantas, su distribución se asocia principalmente a los factores climáticos y edáficos (Hanson y Churchill, 1961; Chapman, 1976).

La amplitud ecológica también se conoce como perfil bioclimático (Fischer et al., 2001; Graham et al., 2004; Vaughton y Ramsey, 2004; Villaseñor y Téllez-Valdez, 2004; Finch et al., 2005) o perfil ecológico (Petraglia y Tomaselli, 2003; Mitov y Stoyanov, 2005). Estos perfiles proporcionan información acerca del intervalo de tolerancia de las especies, lo cual permite evaluar su afinidad respecto a los factores ambientales y hace posible medir su probabilidad de presencia en el espacio geográfico.

Debido a que es determinante para su distribución, el nicho ecológico es fundamental para las especies, que por este motivo generalmente desarrollan mecanismos que les permiten la dispersión de su nicho en el espacio (Townsend, 2001).

El nicho fundamental está delimitado por las condiciones ambientales que requiere una especie y puede estar afectado por la heterogeneidad del paisaje y por algunos factores como la fragmentación, el tamaño de los parches y el efecto de borde. La distribución de una especie también puede estar limitada por la interacción con otras especies, como la competencia interespecífica, la depredación o el parasitismo, así como por interacciones positivas, como simbiosis, y por limitantes a la dispersión (Aráujo y Guisan, 2006). En general, lo anterior ocasiona que su nicho adquiera distribuciones más restringidas, lo cual es conocido como nicho realizado (Hutchinson, 1957; Mazerolle y Villard, 1999; Aráujo y Guisan, 2006; Stockman et al., 2006).

Una reciente aplicación de los Sistemas de Información Geográfica (SIG) en ecología es la construcción de modelos de los nichos fundamentales, con los que es posible predecir la distribución geográfica de las especies a partir de registros de recolección (Townsend y Klusa, 2003). Los modelos predictivos simplifican el concepto de nicho tomando en cuenta los factores ambientales de las localidades de recolección de la especie con intención de modelar el nicho ecológico fundamental (Illoldi-Rangel et al., 2002; Finch et al., 2005). Se han desarrollado diversas técnicas que permiten la construcción de modelos de nicho, principalmente mediante la utilización de información ambiental, de registros de las especies y de parámetros demográficos (Brito et al., 2009; Alba-Sánchez et al., 2010; Scoble y Lowe, 2010).

De acuerdo con Williams et al. (2002), la construcción de modelos de distribución potencial require 3 tipos de información ambiental: $a$ ) terreno, b) clima y c) substrato.
El terreno incluye parámetros como elevación, pendiente, relieve y exposición, los cuales pueden generarse a partir de datos topográficos y su posterior transformación en un modelo digital de elevación (MDE), constituyéndose en uno de los insumos más importantes en la modelación. La información climática siempre es escasa, especialmente cuando se analizan aspectos asociados a la conservación de bienes y servicios ambientales, ya que generalmente, y particularmente cierto para el caso de de México, las estaciones meteorológicas están instaladas en áreas agropecuarias donde los datos se utilizan para evaluar actividades de ese sector (Mendoza, 2002); en consecuencia, el uso de relaciones entre los datos meteorológicos y del terreno son indispensables para la generación de modelos de distribución espacial de parámetros (Leal-Nares et al., 2010). La información de substrato puede ser la más difícil de obtener; sin embargo, hay regiones donde está disponible en forma de bases de datos espaciales de litología, suelos y, en ocasiones, formas del relieve.

Los programas diseñados para la predicción de distribución potencial de especies emplean principalmente aproximaciones de 3 tipos: heurístico estadístico (regresión e interpolación de multivariados) y modelos de computación más complejos, basados en funciones matemáticas (Williams et al., 2002). Es claro que el éxito de estos programas depende de la calidad de los datos ambientales y de los datos de registro de las especies. En la construcción de modelos de distribución potencial de especies, la consolidación de bases de datos biogeográficos digitales es uno de los procesos principales para la creación de los mapas (Jennings, 2000; Williams et al., 2002).

Existen algunas limitantes para el uso de estos programas cuando los organismos están restringidos a parte de su hábitat potencial, ya sea por barreras biogeográficas o por su pobre habilidad de dispersión, que puede verse alterada por acción del hombre (Williams et al., 2002).

Pinus martinezii E. Larsen es una especie que está en la categoría de protección especial en la lista de NOM059-SEMARNAT 2010 (SEMARNAT, 2010), que tiene importancia como especie forestal, y para la cual existen muy pocos trabajos científicos. De acuerdo con una revisión reciente, esta especie se considera sinónima de Pinus durangensis Martínez (Farjon et al., 1997; Farjon y Styles, 1997; Styles, 1998; Ricker y Hernández, 2010); sin embargo, en el presente trabajo se ha reconocido conforme a la clasificación de Larsen (1964) y a los trabajos realizados recientemente por botánicos especialistas en coníferas, quienes difieren en que sean consideradas como la misma especie (Carranza, 1987; Madrigal-Sánchez, 1994; Madrigal-Sánchez y Guiridi-Gómez, 2004; Silva, 2005). Lo anterior se ha basado en la revisión de colecciones científicas almacenadas en la Universidad Michoacana de San Nicolás 
de Hidalgo y en el INECOL, unidad Pátzcuaro. Si bien el propósito de este trabajo no es resolver si taxonómicamente se trata o no de la misma especie, datos sobre la distribución de ésta en Michoacán y más concretamente en la cuenca de Cuitzeo, pueden ser de utilidad para la conservación y adecuado manejo de la misma.

$\mathrm{Al}$ interior de la cuenca de Cuitzeo se han documentado 6 localidades con poblaciones de esta especie (Silva, 2005), todas expuestas a presión por extracción clandestina de madera.

Para generar un mapa de distribución potencial de $P$. martinezii en la cuenca de Cuitzeo se utilizó un análisis multicriterio (AMC) espacialmente explicito. Este análisis se lleva a cabo por medio de un proceso analítico, que constituye un sistema para ayudar a los expertos a mejorar la toma de decisiones mediante el uso de juicios que proveen una estructura y síntesis clara. Es un método flexible que ayuda a analizar problemas de decisión difícil. Consiste en separar de una situación compleja sin estructura, los pequeños componentes que la conforman, asignándoles valores numéricos a partir de juicios subjetivos, para determinar la prioridad de las variables que influyen en una determinada situación (Malczewski, 1997; Al- Zu'bi, 2007). Al igual que en modelos como data driven y knowledge driven, la asignación de pesos en el AMC puede estar basada en criterios estadísticos y/o subjetivos fundados en la opinión de expertos (Bonham-Carter, 1994).

Los mapas de criterios y los pesos finales son integrados para proveer un cálculo final, que se lleva a cabo bajo una apropiada regla de decisión; por ejemplo, una función de agregación lineal. Debido a que la evaluación multicriterio está relacionada con los factores geográficos y sus interacciones, éstos se pueden analizar dentro de un Sistema de Información Geográfica (SIG); así, cada píxel en el espacio se evalúa con base en criterios ponderados para obtener una escala jerárquica de valores de aptitud que pueden representarse en forma de mapas expresando la compatibilidad del territorio (Bonham-Carter, 1994; Paegelow et al., 2003).

En este estudio, se elaboró un modelo de distribución potencial de Pinus martinezii en la cuenca de Cuitzeo, utilizando información ambiental y datos de presencia de la especie, lo que requirió identificar los factores ambientales que determinan el nicho fundamental de P. martinezii y elaborar un perfil bioclimático de la especie. La presente investigación parte de la hipótesis de que el intervalo de tolerancia de P. martinezii a los factores ambientales determina su distribución en el espacio geográfico, por lo que el nicho fundamental de la especie puede ser representado a partir de un modelo de distribución potencial, en el cual sean identificadas las áreas aptas para la conservación y potencial reforestación de sus poblaciones en la cuenca de Cuitzeo.
La construcción del modelo de distribución potencial se basó en la comparación de 3 escenarios apoyados en igual número de técnicas multicriterio generalmente utilizadas, y que son claras para la mayoría de los usuarios. La comparación entre los 3 escenarios fue validada con un índice que incorporó datos de densidad, área basal y frecuencia de los individuos de $P$. martinezii en las distintas localidades. Una vez identificado el mejor modelo de distribución espacial, se identificaron los sitios de distribución potencial. Los productos obtenidos de este proyecto pueden utilizarse con la finalidad de apoyar estrategias de planeación (Brito et al., 2009; Scoble y Lowe, 2010).

\section{Materiales y métodos}

Área de estudio. La cuenca de Cuitzeo está localizada entre los $19^{\circ} 30^{\prime}$ y $20^{\circ} 05^{\prime} \mathrm{N}, 100^{\circ} 30^{\prime}$ y $101^{\circ} 35^{\prime} \mathrm{O}$ (Fig. 1); tiene una superficie de $4000 \mathrm{~km}^{2}$ (Mendoza et al., 2010) que abarca un total de 28 municipios, de los cuales 23 corresponden a la parte norte de Michoacán (92.8\%) y 5 al sur del estado de Guanajuato (7.2\%). La precipitación anual promedio en la cuenca está calculada en $847.4 \mathrm{~mm}$

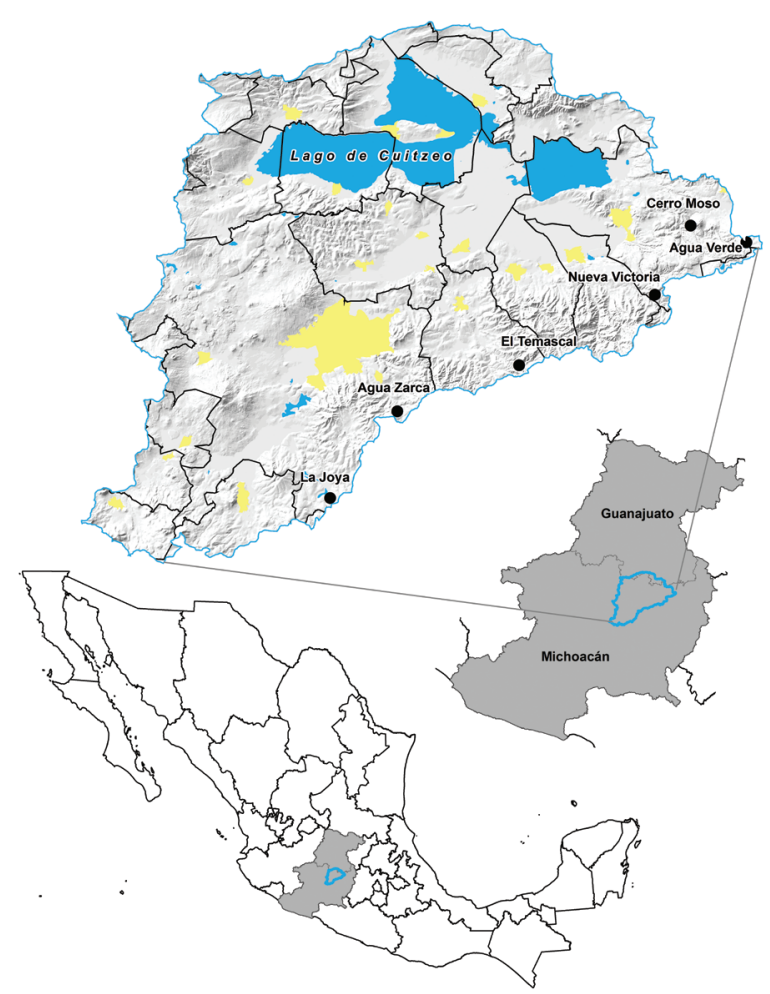

Figura 1. Localización de la cuenca del lago de Cuitzeo, mostrando los sitios de muestreo de Pinus martinezii y la distribución de los límites de los municipios presentes en la cuenca. 
y la temperatura media anual oscila de $16-18^{\circ} \mathrm{C}$ para casi toda el área (Mendoza et al., 2006), con excepción de las tierras altas donde se presentan temperaturas de $14-16^{\circ} \mathrm{C}$. Con base en los criterios establecidos por García (2004), se estimaron 3 tipos climáticos de las estaciones para este trabajo: semicálido, templado y semiseco. Mendoza y Bocco (2010) describen la existencia de 6 zonas geomorfológicas: planicies, piedemontes, colinas, lomeríos bajos, lomeríos altos y montañas. En la cuenca existen 11 unidades principales de suelo de acuerdo con el sistema clasificatorio FAO 1990 (INEGI, 2004) aunque la mayor parte de la cuenca está cubierta por vertisoles, luvisoles y acrisoles, caracterizados por ser suelos con texturas predominantemente finas (Mendoza et al., 2001). De acuerdo con López Granados et al. (2006), la clase de cobertura vegetal dominante son los matorrales con el $24.2 \%\left(958.4 \mathrm{~km}^{2}\right)$ de la superficie total, seguida por los bosques con el $20 \%\left(793 \mathrm{~km}^{2}\right)$ y posteriormente, los usos del suelo, caracterizados por los cultivos de temporal con $19.9 \%\left(782.2 \mathrm{~km}^{2}\right)$ y los cultivos de riego con el $13.31 \%$ $\left(526.6 \mathrm{~km}^{2}\right)$.

La construcción del perfil bioclimático y el modelo de distribución potencial de $P$. martinezii requirió la integración de datos digitales de tipos de roca (escala 1:100 000, Garduño-Monroy y Alcántara, 2010), formas de relieve (escala 1:50 0000; Mendoza y Bocco, 2010) y grupos de suelos (Mendoza et al., 2001; a partir de mapas de 1971, 1973, 1979, 1982 y 1983 escala 1:50 000,), así como topográficos, en formato vectorial, a escala original 1:50 000 de INEGI (1999). Las bases de datos espaciales fueron integradas y rectificadas a proyección UTM (Universal Transversa de Mercator) zona 14, dentro del ArcView 3.2 (ESRI, 1999) y ArcGis 9.2 (ESRI, 2008); todos los objetos con un área mínima cartografiable menor a $3 \times 3 \mathrm{~mm}\left(22500 \mathrm{~m}^{2}\right)$ fueron agregados a su vecino más cercano y en los mapas con formato raster el tamaño de píxel utilizado fue de 20 metros. El análisis de los datos para el perfil bioclimático y la elaboración del modelo de distribución potencial fueron realizados en ILWIS 3.3 (ITC, 2005).

Registro de información en campo. De acuerdo con las exploraciones realizadas por Silva (2005), en la cuenca existen 12 localidades de $P$. martinezii, de las cuales 6 poseen poblaciones poco significativas debido a que su extensión es menor a $22500 \mathrm{~m}^{2}$, que es el área mínima cartografiable considerada en el manejo de información geográfica en el presente trabajo, por lo que no fueron incluidas en los muestreos.

Los 18 sitios de muestreo fueron ubicados utilizando el método de muestreo preferencial llamado comúnmente representativo, basado en suposiciones a priori acerca de las propiedades de la vegetación, reuniendo un número variable de unidades de muestreo para cada localidad que posteriormente se promedian. Este modelo de muestreo se utiliza cuando el investigador requiere cubrir una variación ambiental compleja, tomando las muestras cuando se presentan condiciones de ambientes distintos (Matteucci y Colma, 1982), En este caso se utilizó debido a que fue necesario que los datos representaran la mayor variación ambiental posible para la elaboración del modelo de distribución potencial; el número de sitios de muestreo y la ubicación de los mismos se fijaron en campo. Como factores determinantes se tomaron en cuenta el tamaño de la población y las variaciones ambientales presentes en la zona.

En cada sitio de muestreo se marcaron círculos de $1000 \mathrm{~m}^{2}$ (0.1 ha) con un diámetro de $35.6 \mathrm{~m}$, dirigidos hacia los puntos cardinales y divididos en sectores numerados en el sentido de las manecillas del reloj. En México es común utilizar este método para trabajos forestales; en Michoacán, se utiliza para la evaluación de comunidades de Pinus martinezii (véase Madrigal-Sánchez, 1994; Silva, 2005). En el centro de cada cuadrante se obtuvo la coordenada UTM para generar una base de datos georreferenciada de las poblaciones muestreadas y facilitar su manejo en un SIG para la elaboración de un modelo de distribución potencial (Kearney y Porter, 2004).

Elaboración del perfil bioclimático de Pinus martinezii. Se describieron los principales factores ambientales relacionados con la distribución espacial de la vegetación, entre los que se encuentran el topográfico y el climático (temperatura, precipitación y humedad), así como las características de los suelos (textura, estructura y profundidad) y los aspectos litomorfológicos (rocas, formas de relieve, exposición y pendiente de la ladera). El perfil bioclimático de $P$. martinezii se obtuvo a partir del establecimiento del intervalo de tolerancia a diversos factores ambientales, determinados mediante sobreposición cartográfica de la base de datos de los puntos donde la especie está presente con los mapas temáticos físico geográficos (altitud, roca, relieve, suelos, pendientes y exposición de la ladera, temperatura media anual y precipitación anual), de acuerdo con el enfoque propuesto por Petraglia y Tomaselli (2003).

Se elaboraron cuadros y gráficas de los factores ambientales presentes en cada sitio de muestreo de $P$. martinezii, para determinar el intervalo ecológico y los factores que están incidiendo en su distribución.

Elaboración del modelo de distribución potencial de Pinus martinezii. El modelo de distribución potencial está basado en datos de presencia de la especie y en los datos de las condiciones ambientales prevalecientes en cada localidad, en el supuesto de que representan el nicho realizado de la especie. Se visitaron 6 localidades 
y se establecieron 18 sitios de muestreo, en los cuales se midieron y registraron los parámetros fisonómicos de un total de 247 árboles. Las 6 localidades representan el $50 \%$ de las registradas por Silva (2005). Se consideró un número razonable, dado que al inicio del trabajo se desconocía el número de localidades donde podría encontrarse la especie en cuestión. De cada sitio se anotaron las siguientes mediciones: 1) número de individuos o densidad (abundancia); 2) cobertura de las copas de los árboles; 3) altura de los individuos y 4) diámetro a la altura de pecho (DAP). Por tanto, el modelo está basado en el nicho fundamental de la especie y sólo representa una aproximación de su nicho ecológico (Anderson et al., 2002; Phillips y Dudík, 2008). El modelo de distribución potencial de $P$. martinezii se elaboró en el módulo de Evaluación Espacial Multicrtiterio de ILWIS V.3.3 (ITC, 2005). El AMC, permitió estructurar objetivos, metas, criterios y datos. Posteriormente, se asignaron valores numéricos bajo juicios de valor y también, en este caso, basados en la recopilación de información del trabajo de campo, a fin de determinar las variables que tienen una mayor influencia en la distribución de la especie.

Los criterios utilizados en el análisis fueron organizados en 3 grandes grupos: geopedológico (roca, relieve, suelos), morfométrico (altitud, pendientes y exposición de la ladera) y climáticos (temperatura media anual y precipitación anual). Únicamente se utilizaron datos de presencia de $P$. martinezii, ya que los de ausencia sólo pueden ayudar a obtener mejores resultados si dentro del área de estudio también se cubren las zonas no aptas para la especie, información con la que no se contó (Chefaoui y Lobo, 2008). Los criterios se normalizaron, se priorizaron y finalmente se agregaron. Al normalizarse, los valores de los criterios pierden su dimensión y se vuelven una expresión en términos de "grados de realización del objetivo" (Geneletti, 2005), en este caso, en grados de aptitud para P. martinezii, lo que hace que los criterios sean comparables debido a que no tienen unidad de medida. La priorización sirve para asignar un peso, un valor de importancia relativa a cada criterio con respecto a los otros. Por último, la agregación combina de manera ponderada los valores de los criterios individuales en un puntaje de aptitud total (Geneletti, 2004).

Normalización de los atributos. Para la normalización de los atributos se aplicó el método de categorías (rating) en las tablas de atributos que corresponden a las clases en cada uno de los mapas.

Con este propósito se utilizaron los datos del perfil bioclimático, obteniéndose el número de registros en campo de $P$. martinezii para cada una de las clases. Se asignó valor de 1 para la clase con más registros y de 0 para aquellas donde no existe registro de la especie; posteriormente, los demás valores se asignan en un gradiente de acuerdo con su intervalo (Mendoza y Macoun, 1999; Geneletti, 2007) (Cuadro 1).

Para los 3 factores que están constituidos como una matriz raster de valores continuos (altitud, precipitación y temperatura), se estandarizaron los valores mediante la normalización de combinación en forma de campana, en la cual se da por hecho que la eficiencia (presencia de la especie) es mayor en el valor modal del criterio (Keeney, 1992; Sharifi y Herwijnen, 2004). Los valores utilizados para generar la estandarización de los atributos continuos corresponden a la media estadística (punto de inflexión de la curva) y a los valores mínimos y máximos, obtenidos a partir del perfil bioclimático de la especie.

Ponderación de criterios y subcriterios. La asignación de pesos para los criterios se realizó mediante 2 métodos, ampliamente utilizados en AMC: 1) la comparación por pares (pairwise comparation) que asigna un valor de importancia a los criterios, comparándolos de 2 en 2 (Saaty, 1980), y 2) la asignación de pesos jerárquica (rank sum), en la cual se le otorga el valor más alto a la variable

Cuadro 1. Obtención de los valores normalizados de los atributos mediante el método de categorías con base en los datos obtenidos en el perfil bioclimático

\begin{tabular}{|c|c|c|c|}
\hline Criterio & Atributos & Registros & Valor \\
\hline \multirow{2}{*}{$\begin{array}{l}\stackrel{0}{\varrho} \\
\stackrel{\Xi}{n}\end{array}$} & Luvisoles & 12 & 0.706 \\
\hline & Andosoles & 17 & 1.0 \\
\hline \multirow{6}{*}{ 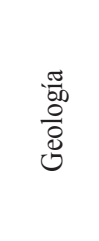 } & Depósitos superficiales & 1 & 0.1 \\
\hline & Depósitos de caída & 2 & 0.2 \\
\hline & Basaltos y dacitas & 4 & 0.4 \\
\hline & Conos andesíticos & 6 & 0.6 \\
\hline & Domos dacíticos y riolíticos & 6 & 0.6 \\
\hline & Ignimbritas & 10 & 1.0 \\
\hline \multirow{4}{*}{ 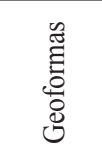 } & Complejos cumbrales de lomeríos altos & 3 & 0.158 \\
\hline & Complejos de laderas de colinas & 3 & 0.158 \\
\hline & Complejos de laderas de montañas & 4 & 0.211 \\
\hline & Complejos de laderas de lomeríos altos & 19 & 1.0 \\
\hline \multirow{4}{*}{ 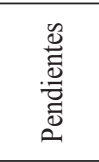 } & $0-4$ & 2 & 0.143 \\
\hline & 4- 8 & 8 & 0.571 \\
\hline & 8- 16 & 14 & 1.0 \\
\hline & $16-25$ & 5 & 0.357 \\
\hline \multirow{9}{*}{ 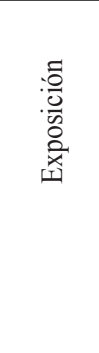 } & Este & 1 & 0.143 \\
\hline & Norte & 1 & 0.143 \\
\hline & Sin exposición $(\mathrm{S} / \mathrm{E})$ & 1 & 0.143 \\
\hline & Suroeste & 1 & 0.143 \\
\hline & Noreste & 4 & 0.571 \\
\hline & Oeste & 4 & 0.571 \\
\hline & Sur & 4 & 0.571 \\
\hline & Sureste & 6 & 0.857 \\
\hline & Noroeste & 7 & 1.0 \\
\hline
\end{tabular}


más importante según la siguiente ecuación (Malczewski, 1999):

$$
W_{k}=\frac{n+1-k}{\sum_{i=1}^{n}(n+1-i)}
$$

Ecuación (1)

donde $W_{k}$ corresponde al peso para el criterio $k, n$ es el número de criterios y $w 1 \geq w 2 \geq \ldots \geq w_{n} \geq 0$.

La asignación de pesos se generó a partir de un taller en el que participaron un botánico, un geógrafo físico y un biólogo de la conservación, los 3 familiarizados con la especie; este tipo de enfoque es el más adecuado para reducir la subjetividad que pudiera involucrar el modelado si no se hace de manera colectiva (Geneletti, 2005; Geneletti, 2009). En dicho taller, se identificaron las variables que tienen una mayor influencia en la distribución de $P$. martinezii. La asignación de pesos a los subcriterios se basó en la técnica jerárquica (rank sum), utilizando los resultados obtenidos en el análisis del perfil bioclimático de Pinus martinezii. Los escenarios de este trabajo son el resultado de la aplicación de diferentes asignaciones de pesos a los criterios, definiendo 3 modelos de salida.

Escenario 1. Este modelo se basó en el método jerárquico, asignándole un mayor peso al criterio geopedológico, seguido por el morfométrico y en última instancia al climático.

Escenario 2. Se efectuó una comparación pareada para la priorización de los criterios, considerándose, en orden de importancia, el geopedológico el altimétrico y el climático.

Escenario 3. Este modelo se realizó mediante la comparación pareada, asignando la misma importancia a los criterios, pero con diferencia en los pesos respecto al escenario anterior.

Se decidió utilizar 3 escenarios, porque se reconoce que en este tipo de modelado existen limitantes para predecir; por lo que se recomienda construir los modelos variando las importancias relativas o los métodos de normalización (Sharifi y Herwijnen, 2004; Mendoza et al., 2008). En este caso se decidió cambiar los métodos de normalización y evaluar la eficiencia de cada modelo.

Los valores normalizados que se utilizaron para la construcción de los 3 modelos de distribución potencial mediante el AMC se resumen en los cuadros 2, 3 y 4. Agregación y obtención del mapa de distribución potencial de Pinus martinezii. Una vez normalizados y priorizados los criterios y subcriterios, se elaboraron los árboles de decisiones para los 3 modelos, asignando los pesos a cada uno de los mapas, a fin de obtener los mapas de distribución potencial de $P$. martinezii, basados en una sumatoria lineal de los pesos, según la ecuación que sigue:

$$
V_{i}=\sum_{i} a_{i} w_{i}
$$

Ecuación (2)

donde $V_{i}$ es el valor de un determinado píxel, $a_{i}$ es el valor del criterio normalizado, $w_{i}$ el peso del criterio, e $i$ es la evaluación del criterio.

El resultado de este proceso es la obtención de mapas potenciales de $P$. martinezii, a partir de las relaciones y los pesos para cada uno de los factores utilizados en el análisis, determinando la aptitud de cada píxel para encontrar las condiciones del nicho ecológico para esta especie. Se obtuvieron 3 diferentes mapas potenciales y se asignaron diferentes pesos a los criterios y subcriterios; posteriormente se realizó una prueba de sensibilidad, tomando en cuenta el criterio experto para elegir el mapa de distribución potencial más adecuado y los valores máximos de píxel (eficiencia) en el mapa.

El modelo potencial resultante fue agregado en 3 clases: muy apto (0.67 a 0.93$)$, medianamente apto $(0.59$ a 0.67$)$ y sin aptitud ( $>0.59)$. Los umbrales para la clasificación en grados de aptitud fueron determinados de las estadísticas del modelo y la curva acumulativa, que se obtuvieron de manera automática mediante el programa ILWIS, donde se estima la eficiencia en el espacio geográfico, representada por píxel para la distribución de la especie en las condiciones ambientales propicias para el nicho ecológico fundamental.

Evaluación de la eficiencia del modelo. En la prueba de confiabilidad se utilizaron 16 sitios de presencia de Pinus martinezii, diferentes a los utilizados en la elaboración del modelo, registrados por Silva (2005) para la cuenca de Cuitzeo. Para realizar esta prueba se efectuó la sobreposición de los puntos con el mapa de distribución potencial.

Para cada localidad de colecta se evaluó un índice análogo al valor de importancia relativa (VIR) que ha sido utilizado ampliamente para comparar la importancia de las especies en una población (Whittaker, 1975), pero que permitiera comparar localidades ponderando la densidad de individuos $(D r)$, el área basal o dominancia $(A B r)$ y la frecuencia $(F r)$ de presencia de $P$. martinezii en cada sitio.

De esta forma, se compararon los resultados obtenidos del VIR para cada una de las localidades, en el supuesto de que cuando 2 o más poblaciones sean comparadas, un valor más alto corresponda a poblaciones de $P$. martinezii con mayor grado de conservación, reflejado principalmente en un número mayor de individuos por hectárea, individuos con una mayor área basal y de 
Cuadro 2. Árbol de decisiones multicriterio del escenario 1

\begin{tabular}{|c|c|c|c|c|c|c|c|c|}
\hline \multicolumn{2}{|c|}{$\begin{array}{c}\text { Peso } \\
\text { (método de } \\
\text { evaluación) }\end{array}$} & \multicolumn{2}{|c|}{ Criterio } & $\begin{array}{c}\text { Peso } \\
\text { (método de } \\
\text { evaluación) }\end{array}$ & \multicolumn{2}{|c|}{ Subcriterio } & $\begin{array}{c}\text { Valor } \\
\text { normalizado } \\
\text { (método) }\end{array}$ & Atributo \\
\hline \multirow{28}{*}{ 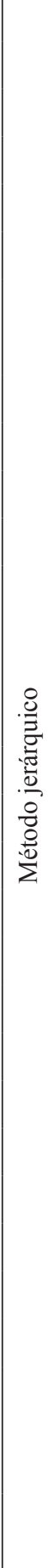 } & \multirow[t]{12}{*}{0.61} & & \multirow{12}{*}{ 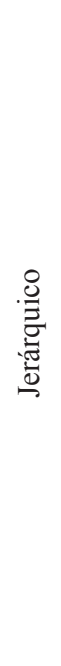 } & \multirow[t]{2}{*}{0.61} & \multirow[t]{2}{*}{ Suelos } & \multirow{12}{*}{ 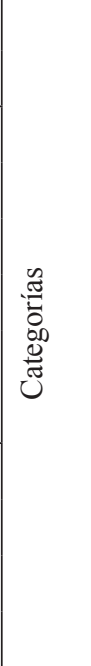 } & 1.00 & Andosoles \\
\hline & & & & & & & 0.71 & Luvisoles \\
\hline & & & & 0.28 & Geología & & 1.00 & Ignimbritas \\
\hline & & & & & & & 0.6 & Conos andesíticos \\
\hline & & & & & & & 0.6 & Domos dacíticos y riolíticos \\
\hline & & & & & & & 0.4 & Basaltos y dacitas \\
\hline & & & & & & & 0.2 & Depósitos de caída \\
\hline & & & & & & & 0.1 & Depósitos superficiales \\
\hline & & & & 0.11 & Geoformas & & 1.00 & Complejos de laderas de lomeríos altos \\
\hline & & & & & & & 0.21 & Complejos de laderas de montañas \\
\hline & & & & & & & 0.16 & Complejos cumbrales de lomeríos altos \\
\hline & & & & & & & 0.16 & Complejos de laderas de colinas \\
\hline & \multirow[t]{14}{*}{0.28} & & \multirow{14}{*}{ 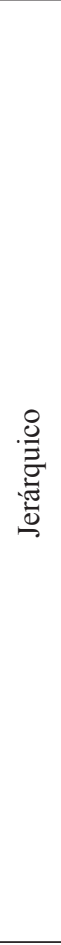 } & 0.50 & Altitud & 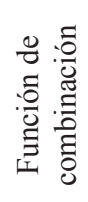 & & \\
\hline & & & & 0.33 & Pendientes & \multirow{13}{*}{ 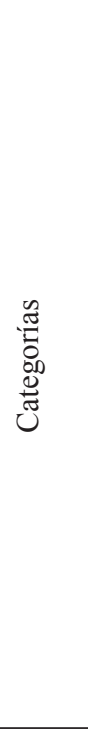 } & 1.00 & 8- 16 \\
\hline & & & & & & & 0.57 & 4- 8 \\
\hline & & & & & & & 0.36 & $16-25$ \\
\hline & & & & & & & 0.14 & $0-4$ \\
\hline & & & & 0.17 & Exposición & & 1.00 & Noroeste \\
\hline & & & & & & & 0.86 & Sureste \\
\hline & & & & & & & 0.57 & Noreste \\
\hline & & & & & & & 0.57 & Oeste \\
\hline & & & & & & & 0.57 & Sur \\
\hline & & & & & & & 0.14 & Este \\
\hline & & & & & & & 0.14 & Norte \\
\hline & & & & & & & 0.14 & $\mathrm{~s} / \mathrm{e}$ \\
\hline & & & & & & & 0.14 & Suroeste \\
\hline & \multirow[t]{2}{*}{0.11} & \multirow{2}{*}{ 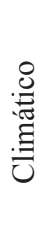 } & \multirow{2}{*}{ 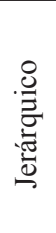 } & 0.50 & Precipitación & \multirow{2}{*}{ 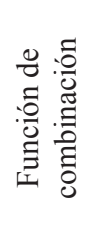 } & & \\
\hline & & & & 0.50 & $\begin{array}{l}\text { Temp. media } \\
\text { anual }\end{array}$ & & & \\
\hline
\end{tabular}


Cuadro 3. Árbol de decisiones multicriterio del escenario 2

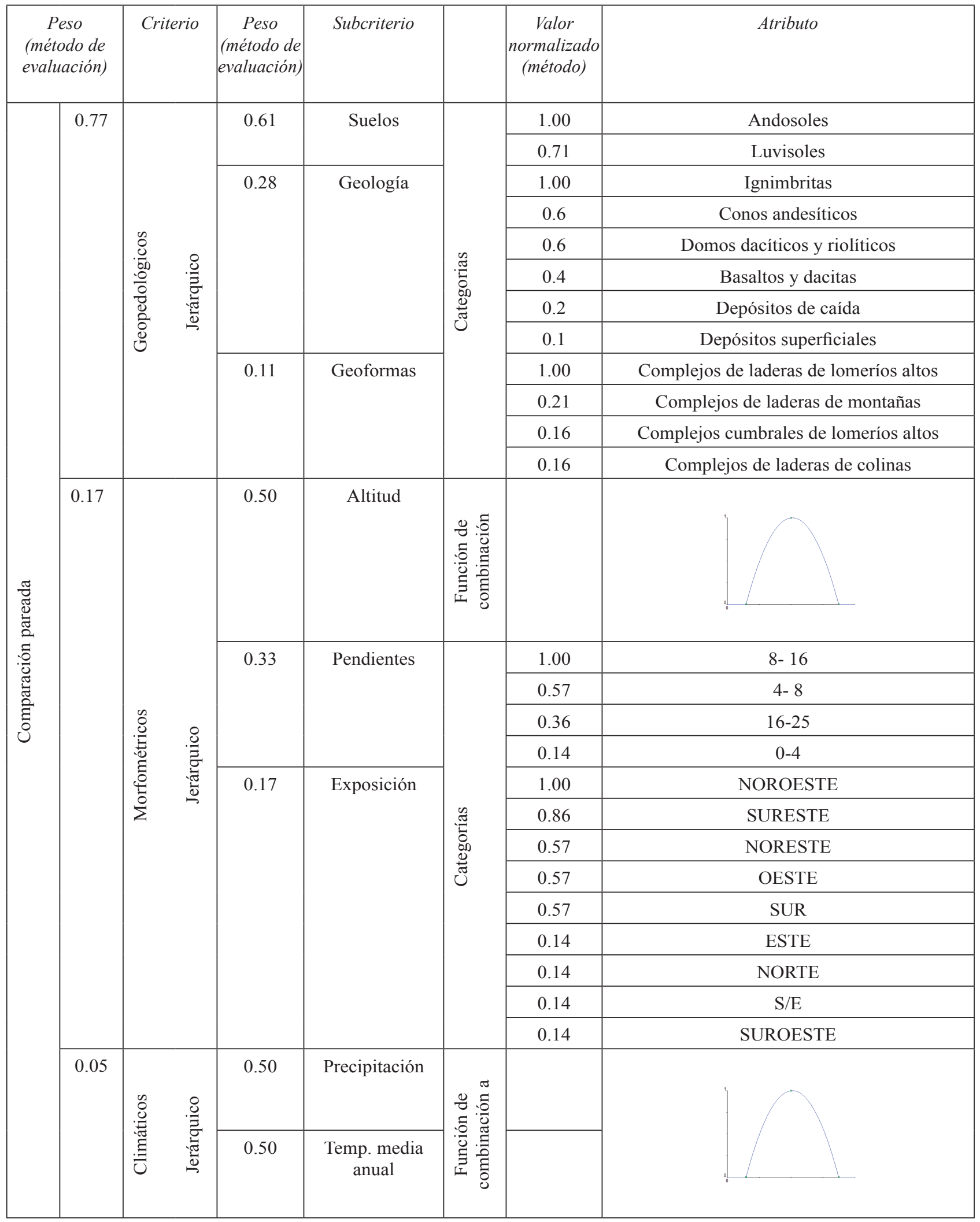


Cuadro 4. Árbol de decisiones multicriterio del escenario 3

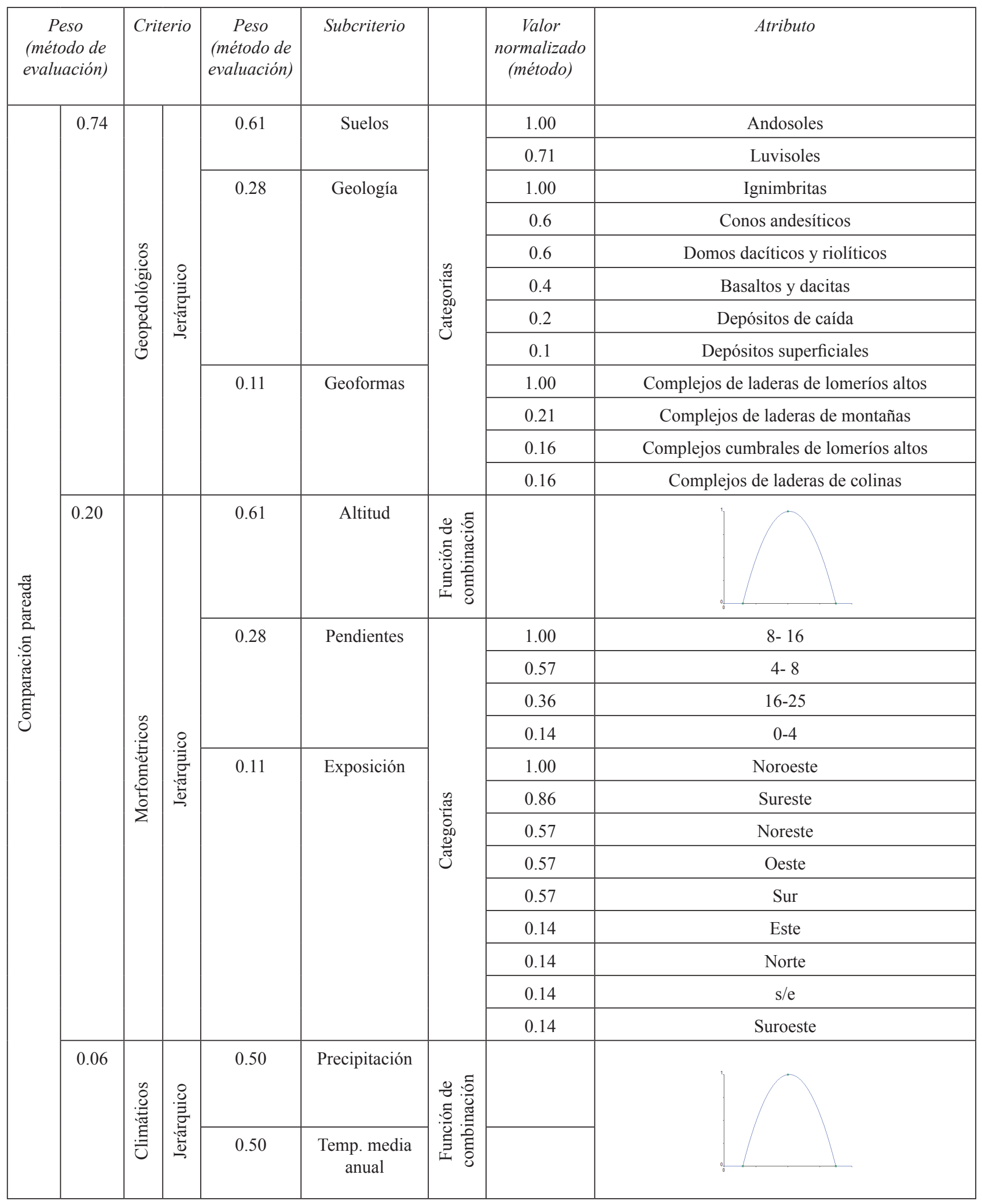


cobertura de la copa, y con una frecuencia mayor de presencia de la especie. En este sentido, las localidades que han sufrido el impacto de disturbios, como incendios, enfermedades, tala para extracción de madera o cambio de uso del suelo, tendrán valores de VIR más bajos. El VIR puede expresarse como porcentaje de importancia de acuerdo con la siguiente ecuación (Curtis, 1959; Matteucci y Colma, 1982):

$$
V I R=\frac{D r+F r+A B}{3}
$$

Ecuación (3)

Identificación de zonas potenciales de conservación y reforestación. La identificación de zonas para conservación y reforestación con $P$. martinezii requirió de la comparación del mapa de distribución potencial con los mapas de cobertura y uso del suelo para la cuenca de Cuitzeo de los años de 1975 y 2000, elaborados por López-Granados et al. (2006). Para este proceso, en el mapa sólo se consideraron las áreas muy aptas para la especie.

Las zonas donde disminuyó la cobertura de la clase bosque de pino y bosque mixto, así como las que presentaron una pérdida o transición a la clase matorral, pastizal o suelo desnudo, se identificaron como áreas potenciales para la reforestación con P. martinezii. El modelo conceptual de este procedimiento se presenta en la figura 2 .

Las áreas susceptibles para la conservación de $P$. martinezii o de presencia probable fueron los bosques de pino cerrado y bosque mixto cerrado que mantuvieron permanencia en la cobertura y los bosques que presentaron coberturas semiabiertas y abiertas. El modelo conceptual del proceso se presenta en la figura 3 .

\section{Resultados}

Perfil bioclimático de Pinus martinezii. Altitud. El intervalo en el que se registró la especie fue de 2 284-3 022 metros. Aunque la distribución fue continua a lo largo de este gradiente, el número de sitios con presencia de $P$. martinezii mostró un patrón bimodal, con el $37.9 \%$ de los sitios registrados entre $2300-2400 \mathrm{~m}$ y el $20.7 \%$ entre los 2 500-2 600 m (Fig. 4).

Suelos. Los sitios con presencia de P. martinezii se restringieron solamente a andosoles (58.6\% de los sitios) y luvisoles (41.4\%) (Fig. 4).

Relieve. Los sitios registrados se ubicaron en 4 clases geomorfológicas; la mayoría $(65 \%)$ coincidió con los complejos de laderas de lomeríos altos, mientras que el $35 \%$ restante se distribuyó de manera marginal y similar en complejos de laderas de montañas, complejos de laderas de colinas y en complejos de cumbres de lomeríos altos (Fig. 4).

Rocas. Pinus martinezii se registró sobre 6 clases diferentes de rocas. Las poblaciones coincidieron con ignimbritas $(34.5 \%)$, domos dacíticos y riolíticos (20.7\%), y conos andesíticos (20.7\%); mientras que en los depósitos superficiales sólo se presentó en el 3.5\% de los sitios (Fig. 4).

Pendiente de ladera. Pinus martinezii se distribuye desde superficies planas hasta laderas con pendientes por encima de los $23^{\circ}$. El $48 \%$ de los sitios se registró en pendientes moderadas, que van de 8 a $16^{\circ}$, seguido por los ubicados en pendientes ligeras, con inclinación de 4 a $8^{\circ}(27.6 \%)$. De acuerdo con los datos analizados puede considerarse que este factor no resulta restrictivo de la distribución; sin embargo, con base en los intervalos de tolerancia de la especie, los valores extremos de este factor pueden afectarla y ocasionarle estrés fisiológico, de manera que sólo pueda mantener algunos individuos debido a la interacción con otras especies, como la competencia, la depredación o el parasitismo (Hutchinson, 1957; Cox y Moore, 1994) (Fig. 4).

Exposición de laderas. Los registros de los sitios comprenden todas las clases existentes en este factor, además de que no muestran un patrón definido, por lo que no se le considera determinante en la distribución de la especie. Pinus martinezii se localizó principalmente en laderas con exposición noroeste (24.1\%) y sureste (20.7\%) (Fig. 4).

Precipitación anual. En los sitios muestreados, $P$. martinezii se localiza desde los 1013 hasta $1499 \mathrm{~mm}$ de precipitación anual; la mayoría se ubica en los valores más bajos, donde la variación en la precipitación oscila entre 1000 y $1050 \mathrm{~mm}$ (44.8\%), mientras que el resto se distribuye casi en forma homogénea en las zonas de mayor humedad (Fig. 4).

Temperatura media anual. En este caso se registra un intervalo de tolerancia de 11.6 a $15.54^{\circ} \mathrm{C}$, y la mayoría de las poblaciones analizadas se distribuyen en zonas con temperatura media anual de 15 a $15.4^{\circ} \mathrm{C}$ (Fig. 4).

Modelo de distribución potencial de Pinus martinezii. El escenario 1 fue descartado debido a que se presentaron valores de aptitud muy bajos en el área correspondiente a la población de Agua Zarca, la cual es la segunda en importancia en la cuenca de Cuitzeo de acuerdo con el VIR (Fig. 5).

En el escenario 2, los valores máximos de aptitud obtenidos (0.63) fueron los menores de los 3, por lo que también fue descartado; sin embargo, cabe señalar que los valores máximos se localizan en las poblaciones de mayor importancia de P. martinezii en el área de estudio (Fig. 5). 
AREAS CON LAS CONDICIONES ECOLÓGICAS APTAS PARA EL DESARROLLO DE Pinus martinezii

\begin{tabular}{|c|c|c|}
\hline COBERTURA AÑO 1975 & & COBERTURA AÑO 2000 \\
\hline \multirow{2}{*}{$\begin{array}{l}\text { Bosque de pino cerrado } \\
\text { Bosque de pino semiabierto } \\
\text { Bosque de pino abierto } \\
\text { Bosque mixto cerrado } \\
\text { Bosque mixto semiabierto } \\
\text { Bosque mixto abierto }\end{array}$} & $\begin{array}{c}\text { CAMBIO EN LA DENSIDAD } \\
\text { O } \\
\text { PERMANENCIA DEL BOSQUE } \\
\text { ABIERTO Y SEMIABIERTO }\end{array}$ & $\begin{array}{l}\text { Bosque de pino semiabierto } \\
\text { Bosque de pino abierto } \\
\text { Bosque mixto semiabierto } \\
\text { Bosque mixto abierto }\end{array}$ \\
\hline & PÉRDIDA & $\begin{array}{l}\text { Matorral- pastizal abierto } \\
\text { Matorral- pastizal semiabierto } \\
\text { Matorral cerrado } \\
\text { Pastizal } \\
\text { Suelo desnudo }\end{array}$ \\
\hline
\end{tabular}

Figura 2. Áreas aptas para la reforestación con Pinus martinezii.

\section{AREAS CON LAS CONDICIONES ECOLÓGICAS APTAS PARA EL DESARROLLO DE Pinus martinezii}

\section{COBERTURA AÑO 1975}

Bosque de pino cerrado Bosque mixto cerrado

Bosque de pino semiabierto Bosque de pino abierto Bosque mixto semiabierto Bosque mixto abierto

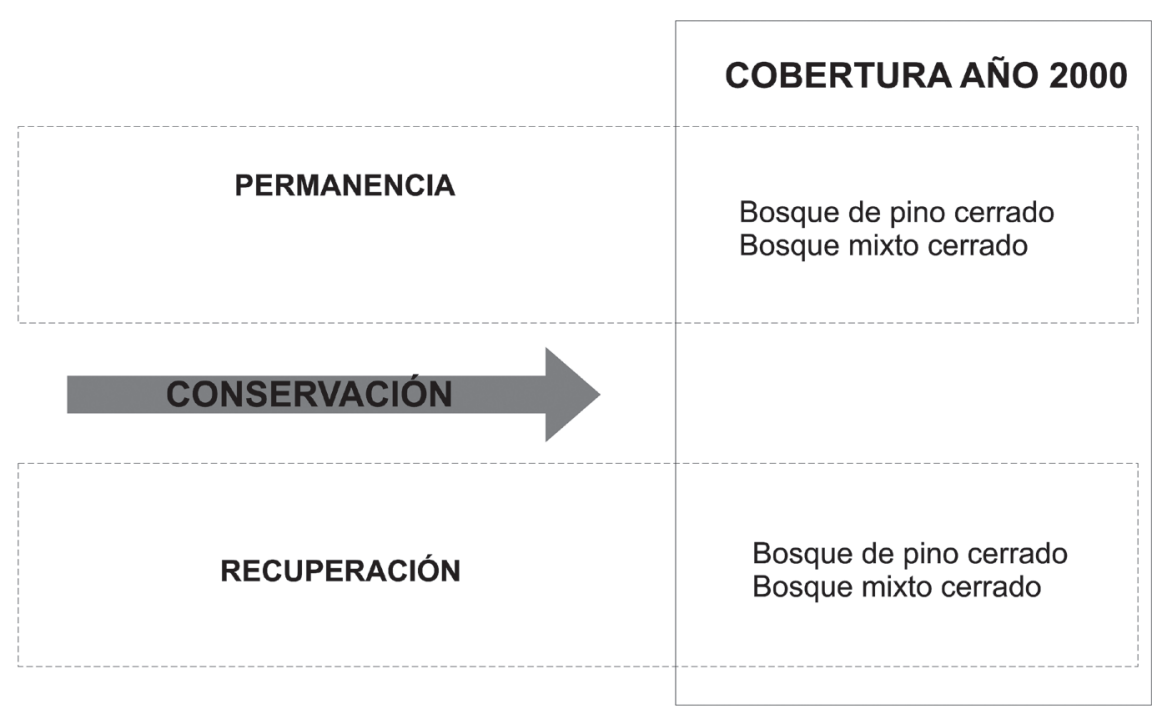

Figura 3. Áreas aptas para la conservación de Pinus martinezii.

Por último, el escenario 3 presentó el valor más alto de aptitud con 0.93 (Fig. 5), cubriendo las poblaciones de mayor importancia de P. martinezii con relación al VIR, lo que es un indicador de que están presentes las condiciones ambientales en la zona para la distribución de la especie.
Por lo tanto, este mapa fue considerado para la obtención del modelo de distribución potencial final (Fig. 6).

El mapa de distribución potencial muestra que el área de aptitud alta con mayor extensión corresponde a la porción oriente de la cuenca, que comparten los municipios 


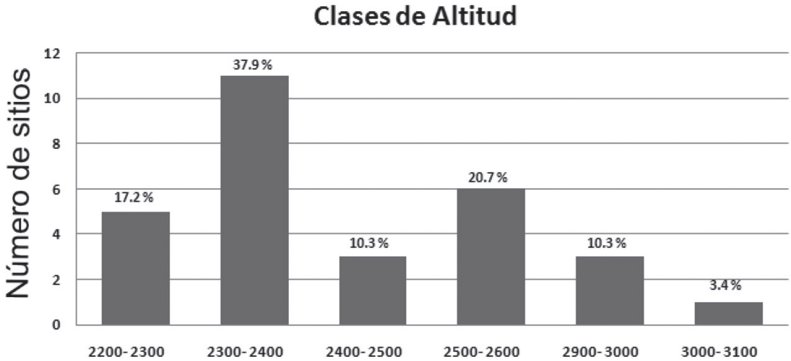

Clases de Precipitación

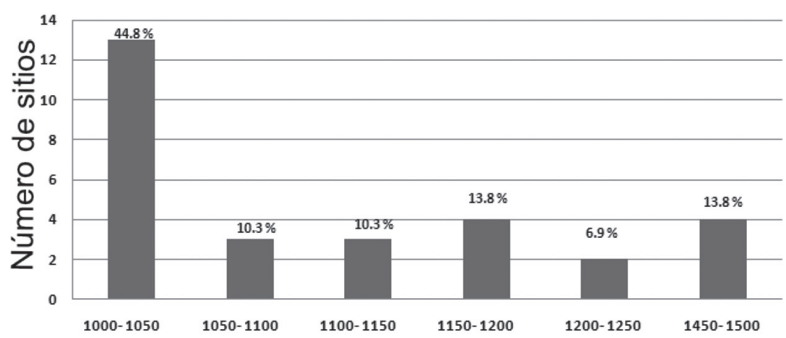

Clases de Temperatura

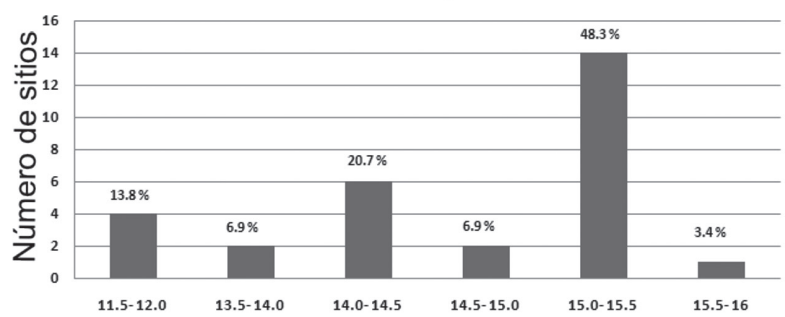

Clases de Pendientes de Laderas

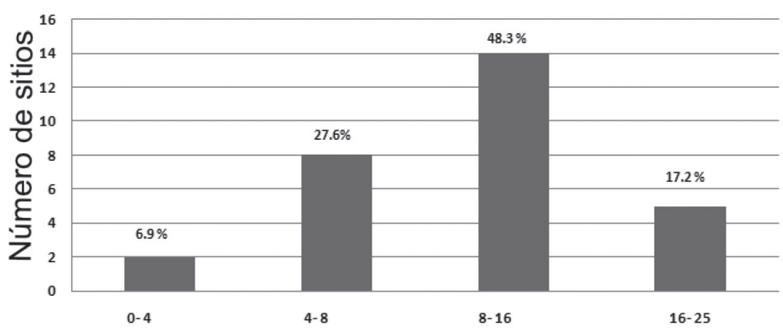

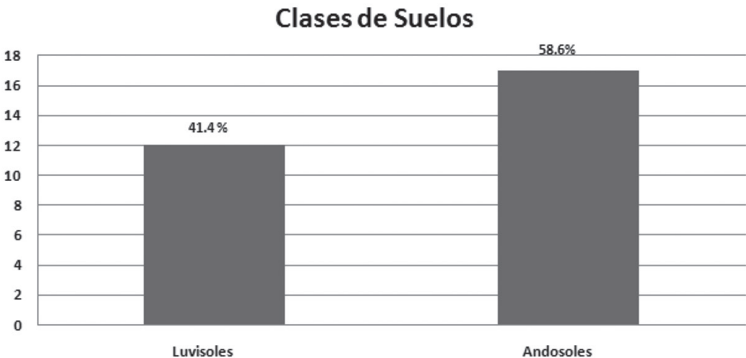

Clases de Exposición de ladera

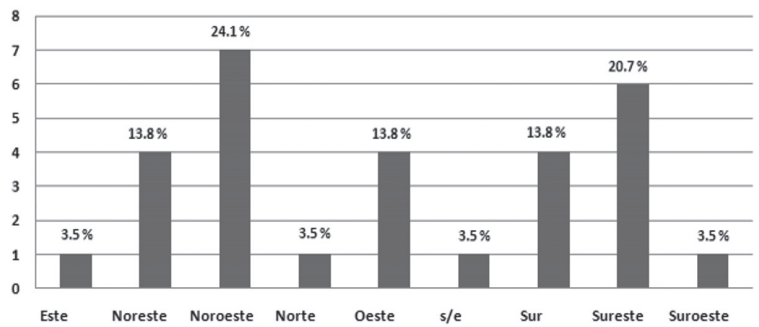

Cases de Rocas

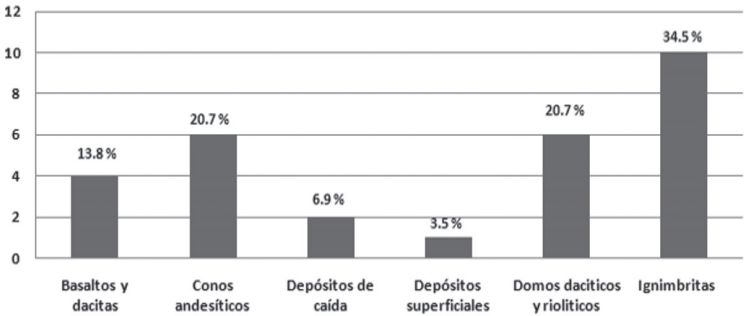

Clases de Relieve

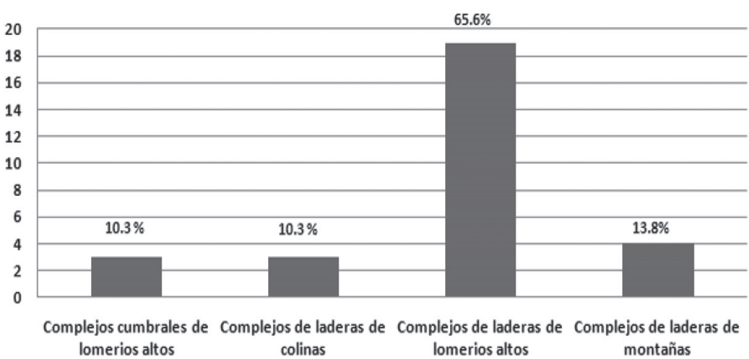

Figura 4. Distribución de los sitios registrados en campo de Pinus martinezii en la cuenca del lago de Cuitzeo por clases.

de Zinapécuaro e Hidalgo. En esta zona se encuentran las localidades de Nueva Victoria, Laguna Verde y Cerro Mozo, la primera considerada como la más importante de acuerdo con el VIR. En esta área es mayor la proporción de zonas de aptitud alta en relación con las de aptitud media.

En el mapa de distribución potencial, Nueva Victoria se ubica en un área con aptitud alta para el desarrollo de $P$. martinezii, lo que concuerda con el VIR que se determinó con base en los registros de campo, ya que se observó que se efectúan labores de conservación por parte de pobladores de ejido, lo que favorece la obtención de un valor de importancia alto.

En el caso de Laguna Verde localizada en Los Azufres, es importante señalar que no fue una de las localidades con VIR más alto, debido a la baja densidad de individuos ocasionada por la extracción de madera; sin embargo, según el modelo de distribución potencial, existen las condiciones ambientales adecuadas para un desarrollo 

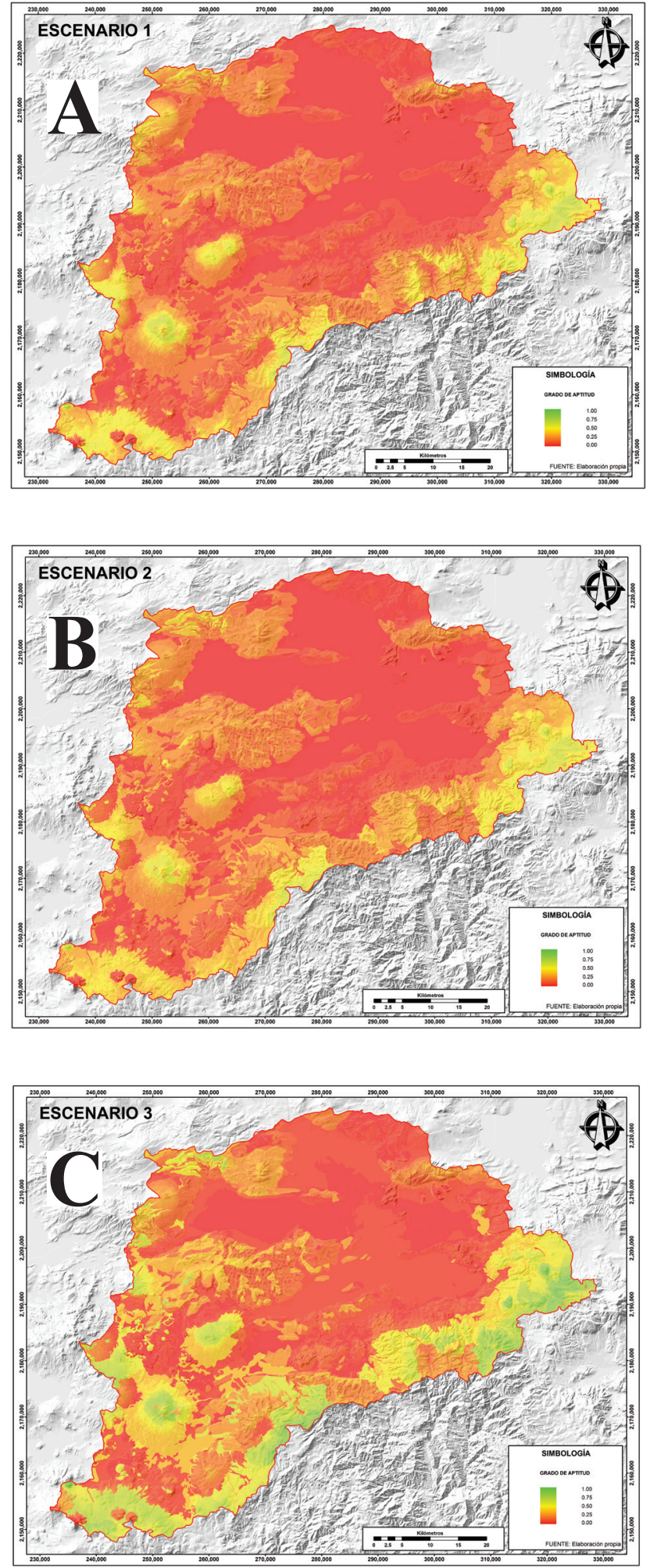

Figura 5. Mapas de distribución potencial de Pinus martinezii obtenidos a partir del AMC para los escenarios 1, 2 y 3 . óptimo de las poblaciones de P. martinezii; lo anterior fue constatado en campo al registrarse los individuos con DAP más elevado.

En el Cerro Mozo se encuentran zonas aptas cercanas a los sitios de muestreo y medianamente aptas en la parte alta de esta estructura, donde no se observaron árboles de esta especie en las excursiones realizadas. Años atrás, las condiciones de esta localidad fueron afectadas por un incendio que casi acabó con del bosque, por lo que hoy sólo pueden encontrarse individuos aislados de $P$. martinezii.

La segunda zona de importancia para la distribución de la especie es la porción sureste del municipio de Morelia, donde se encuentra la localidad de Agua Zarca, segunda en condiciones de conservación de acuerdo con el VIR, formando un corredor en la parte alta de las Calderas La Escalera y Atécuaro. Hacia el sur de la cuenca se encuentran áreas aisladas de aptitud media, que pueden representar un potencial para el hallazgo de nuevas poblaciones de $P$. martinezii.

Confiabilidad del modelo. De las 13 localidades que fueron consideradas por el modelo, 12 se encuentran en la clase "aptitud alta"; sólo Milpillas pertenece a "aptitud media". Estos resultados indican que se obtuvo un $81.25 \%$ de confiabilidad en el modelo, porcentaje que se considera aceptable para un modelo de distribución potencial (Stockwell y Townsend, 2002).

A partir del índice de valor de importancia relativa (VIR) se obtuvo un parámetro estimativo de las condiciones ecológicas de las poblaciones de P. martinezii, mediante el cual Nueva Victoria presentó el valor más alto (30.48), seguido por Agua Zarca (30.40) y en última instancia La Joya (7.09). Los valores obtenidos mediante el cálculo de este índice reflejan las condiciones observadas en los recorridos de campo, siendo las de Nueva Victoria y Agua Zarca las que muestran mayor grado de conservación, mientras que la población localizada en La Joya presentó disturbios por la extracción de madera (Cuadro 5).

\section{Discusión}

Cabe destacar que a diferencia de varios trabajos previos (véase entre otros, Illoldi-Rangel et al., 2004; Kearney y Porter, 2004), en éste se modeló la distribución de una especie de pino con datos de mayor resolución espacial y se validó el resultado utilizando un símil del valor de importancia relativa. Los mapas de distribución potencial elaborados a partir de la aplicación de técnicas de análisis espacial implantadas en un SIG han demostrado ser bastante confiables, en la medida en que proporcionan una aproximación razonable al nicho de las especies, incluso sin la presencia de muchos puntos (Chefaoui et al., 2005). 


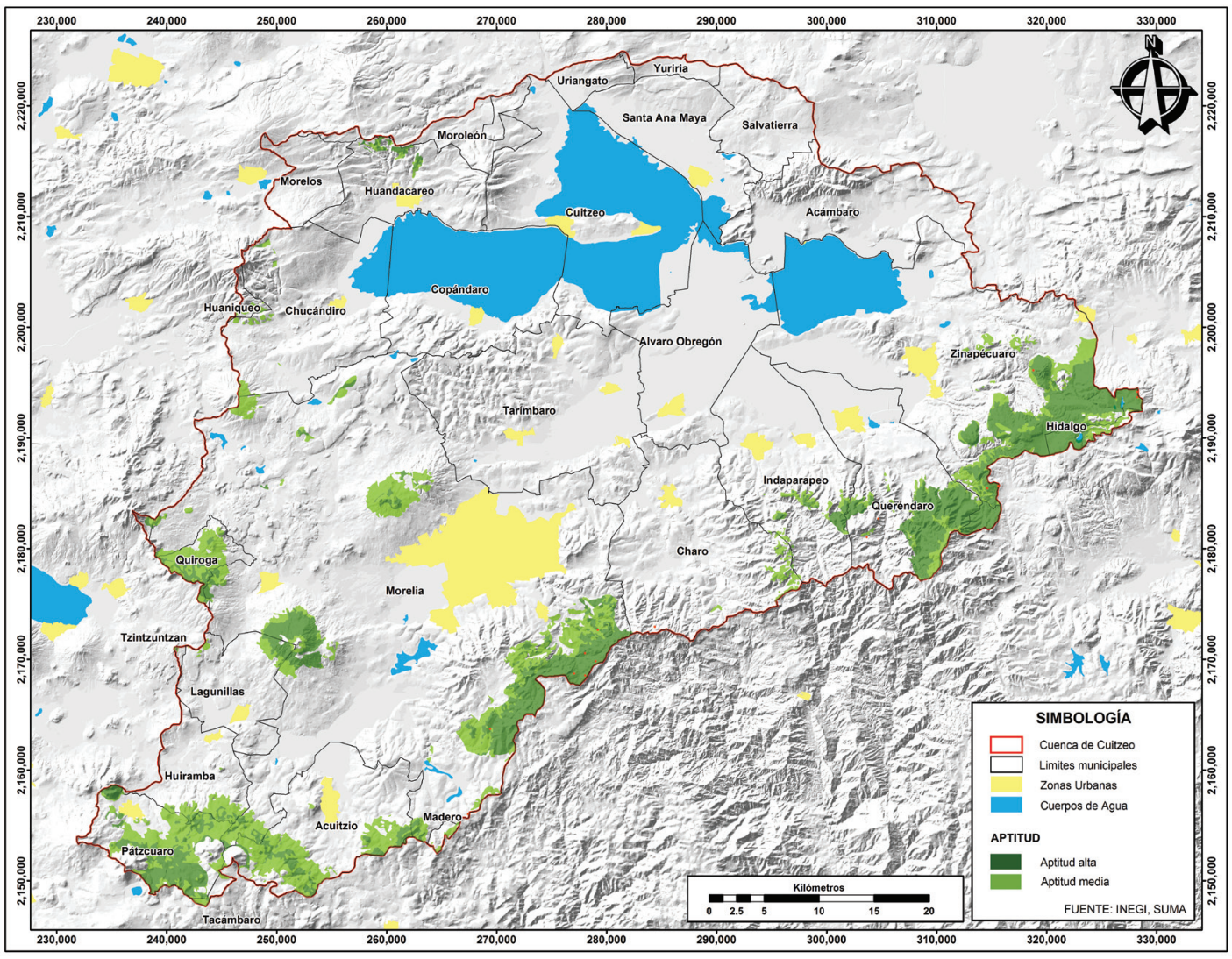

Figura 6. Mapa de clases de aptitud de la distribución potencial de Pinus martinezii basado en el escenario 3.

En el presente trabajo, el escenario más adecuado fue el que se elaboró a partir de comparaciones pareadas con el procedimiento de análisis jerárquico de Saaty (1980), ampliamente utilizado en AMC.

En este caso, el modelo requirió reconocer las relaciones observadas entre factores biofísicos mediante una técnica de análisis multicriterio, más que multivariante. El análisis multicriterio espacialmente distribuido y la resolución de los datos permitieron identificar unidades espaciales más continuas y no referidas a píxeles relativamente aislados. Tomando en consideración la disponibilidad y detalle de los datos existentes, el enfoque utilizado en este trabajo puede reproducirse a escalas menores, a escala de provincia fisiográfica o de país, no sólo en México, sino en muchos otros países.

Es necesario tomar en cuenta que el método utiliza únicamente datos de presencia de la especie y el error de omisión sólo se cuantificó. Las áreas en las que se predice la presencia de la especie en los modelos de distribución potencial son típicamente mayores que sus distribuciones reales, debido a diversos factores que impiden su dispersión, como son las barreras geográficas, las interacciones bióticas y las modificaciones humanas al ambiente, por lo que pocas especies ocupan todas las áreas que satisfacen los requerimientos de su nicho. En dado caso, si fuera necesario cartografiar la distribución realizada de una especie a partir de un modelo de distribución potencial, sería a través de la remoción de áreas en las que se infiere que no puede habitar la especie, sea por factores geográficos o por tratarse de zonas inhabitadas por las interacciones bióticas.

Los modelos de distribución potencial pueden ser una herramienta muy importante para inferir la distribución actual para propósitos de conservación, al compararse con las coberturas y uso del suelo con intención de excluir áreas altamente alteradas y deforestadas.

Zonas potenciales para reforestación con Pinus martinezii. Se detectaron las zonas para reforestación tomando como base la zona de aptitud más alta del modelo de distribución potencial. La diferencia entre los mapas de reforestación obtenidos y lo que se registra en campo se debe a que el último insumo para realizar el mapa de cambio de cobertura es del año 2000. En la mayoría de las localidades visitadas, la extracción de madera clandestina es reciente y se presenta de manera acelerada, no existe registro de tal en dicha cartografía y por lo tanto no aparece en el mapa 
de zonas potenciales para reforestación. Sin embargo, en el presente trabajo se hace énfasis en ello, como un aspecto a tomarse en cuenta para llevar acciones de reforestación. En todas las localidades visitadas la extracción de madera existe en diferentes grados, y dado el carácter de protección especial que tiene la especie, su aprovechamiento debería estar inmerso en un manejo integral que asegure la adecuada regeneración de los ecosistemas donde se encuentra. Es claro que debido a la extracción clandestina en varias de las localidades esto no está ocurriendo.

Los resultados obtenidos mostraron que por sus condiciones de deterioro, las localidades de Cerro Mozo y Laguna Verde son las que requieren de acciones de reforestación. En la porción oriente de la cuenca, donde se ha perdido cobertura de bosque, también existen diversas áreas que presentan las condiciones ecológicas apropiadas para el desarrollo de la especie con base en el nicho ecológico fundamental.

En Cerro Mozo es necesaria una reforestación; sin embargo, hay que tomar en cuenta otros factores, como la pérdida de suelo por la erosión, que puede ser determinante en el establecimiento de un programa de esta naturaleza en este sitio. Como son pocos los individuos de P. martinezii que se encontraron, podrían utilizarse semillas de Nueva Victoria, localidad cercana cuyo perfil bioclimático es similar al de Cerro Mozo; presentó el VIR más alto, lo que indica las buenas condiciones fisonómicas de los individuos del lugar.

En Laguna Verde se detectó una zona susceptible de reforestación. Sin embargo, durante el recorrido de campo en toda el área, fueron pocos los individuos que pudieron localizarse, por lo que se considera necesario llevar a cabo acciones inmediatas. La región presenta condiciones muy favorables para el establecimiento de $P$. martinezii, y de no contarse con los suficientes individuos en el área para formar un banco de semillas, puede recurrirse a la población de Nueva Victoria.

La localidad de Temascal no fue representada en el modelo de distribución potencial y por lo tanto tampoco en el mapa de zonas de reforestación, pero es necesaria una reforestación con la especie, pues en los recorridos de campo se pudo observar que la población de este lugar está sujeta a una tala selectiva que deja muy pocos individuos en pie para una regeneración natural. Debido a su lejanía geográfica con respecto a los demás sitios, lo que también refleja sus diferencias en las condiciones ambientales, esta localidad pudiera enfrentarse a un problema. No obstante, se puede considerar que existe una afinidad con el perfil ecológico de Nueva Victoria, por lo que podría ser un referente para el establecimiento de una plantación.

La localidad de La Joya tampoco fue representada en el modelo de distribución, pero debido a que presentó graves disturbios por extracción de madera, que incluso impidieron el registro de información en el momento de los recorridos de campo, se recomienda realizar una reforestación con $P$. martinezii basada en el sitio de colecta. No se cuenta con suficiente información para determinar si existe material para el establecimiento de un banco de semillas en esta localidad; en dado caso, puede utilizarse germoplasma de Agua Zarca por su cercanía y similitud ecológica. En las zonas cercanas se ubicaron individuos aislados de $P$. martinezii, lo que indica que quizá fue una población que presentaba continuidad con la de Agua Zarca. Tomando en cuenta lo anterior, se recomienda utilizar semillas provenientes de Agua Zarca, que presentó un VIR alto debido a sus condiciones ecológicas. Por otro lado, en esta localidad existen manchones aislados potenciales para la reforestación, lo que es un indicativo de las condiciones de conservación del lugar.

El modelo de distribución potencial considera áreas aisladas donde no se registraron poblaciones de P. martinezii, sobre todo en la porción norte y poniente de la cuenca, lo que indica que aunque en apariencia hay condiciones para el desarrollo de poblaciones, éstas no se encuentran. Debido a los alcances de este proyecto, no es posible saber si dicha ausencia se deba a factores naturales, tales como la presencia de barreras biogeográficas que impidan la colonización de P. martinezii, a alguna interacción ecológica que excluya a esta especie o la ausencia de alguna otra que facilite su establecimiento (Aráujo y Guisan, 2006), o bien, a factores

Cuadro 5. Índice de Valor de Importancia Relativa (VIR) por localidad

\begin{tabular}{lcccc}
\hline Localidad & Área basal relativa & Densidad relativa & Frecuencia relativa & Valor de importancia relativa \\
\hline Nueva Victoria & 27.89 & 40.86 & 22.71 & 30.48 \\
Agua Zarca & 39.88 & 29.57 & 21.74 & 30.40 \\
Temascal & 14.05 & 6.74 & 18.12 & 12.97 \\
Cerro Mozo & 3.90 & 10.89 & 15.22 & 10.00 \\
Laguna Verde & 11.33 & 5.71 & 10.14 & 9.06 \\
La Joya & 2.96 & 6.23 & 12.08 & 7.09 \\
\hline
\end{tabular}


asociados al uso del suelo en el pasado, como las prácticas inadecuadas de aprovechamiento forestal o el cambio de cobertura del suelo.

Zonas potenciales para la conservación de Pinus martinezii. Las zonas que aparecen como áreas de conservación son bosques que han permanecido o se han recuperado y que presentan una aptitud alta, por lo que también puede realizarse reforestación en caso de existir un cambio de uso del suelo o incluso el establecimiento de una plantación con fines forestales. También indican una probabilidad de encontrar poblaciones de P. martinezii en futuras exploraciones, ya que existen las condiciones ambientales adecuadas para su desarrollo.

Entre las localidades de muestreo que aparecen como áreas aptas para la conservación se encuentran Agua Zarca, Nueva Victoria, Cerro Mozo y Laguna Verde. Las 2 primeras presentan condiciones estables, por lo que para su mantenimiento es suficiente con un buen manejo orientado al aprovechamiento sustentable, lo que concuerda con lo observado en campo y con el VIR. Cerro Mozo y Laguna Verde presentan rodales de conservación; sin embargo, es necesario realizar un trabajo a mayor detalle pues estas zonas han sido sujetas a disturbios en años recientes.

Los perfiles bioclimáticos generaron información que permite conocer aspectos sobre los intervalos de tolerancia de $P$. martinezii hacia los factores físico geográficos y en esta forma determinar la manera en que éstos influyen en su distribución. Lo anterior constituye una herramienta de utilidad para efectuar acciones de reforestación y sitios de colecta de semilla para el establecimiento de viveros. El modelo de distribución potencial predice la aptitud de un punto en el espacio para $P$. martinezii en función de las variables ambientales utilizadas. El modelo de distribución potencial representa el nicho fundamental de la especie, que consiste en un conjunto de condiciones que le permiten sobrevivir; por lo tanto, el nicho realizado o distribución actual puede ser menor y ocupar sólo una parte del nicho fundamental. Esta diferencia puede deberse a diversos factores entre los que se encuentran: 1) la influencia humana, como el aprovechamiento, el cambio de uso del suelo y los incendios; 2) las interacciones bióticas, como la competencia interespecífica con otros pinos y encinos, y 3) las barreras geográficas, como la presencia de planicies en la cuenca y las diferentes formaciones geológicas que han impedido la dispersión y colonización de nuevas áreas, limitando su distribución.

En este estudio se construyeron 3 escenarios de distribución basados en diferentes árboles de decisión multicriterio y los valores más altos de aptitud fueron mostrados por el escenario 3 que a su vez presentó un $81.25 \%$ en la prueba de confiabilidad.
En el mapa de nicho ecológico fundamental de $P$. martinezii se pueden observar 2 regiones de distribución importantes; en la primera, localizada en la porción oriente de la cuenca, se encuentran 3 de las localidades estudiadas; la segunda, al sur del municipio de Morelia, está constituida por 1 localidad estudiada y otras que no fueron registradas en el presente trabajo debido a que el tamaño poblacional no es significativo. Entre estas 2 zonas de distribución se muestra un área sin aptitud para la distribución de la especie, la cual puede ser atribuida a factores geopedológicos (roca, relieve y suelos).

En la porción sur y este de la cuenca se modelaron áreas de aptitud media para P. martinezii; sin embargo, no existen registros de la especie, lo que indica que existen las condiciones ambientales propicias para el establecimiento de la especie, pero que existen barreras que han impedido su distribución.

La información generada en el perfil bioclimático de $P$. martinezii fue de utilidad al efectuar el AMC para modelar en los SIG el nicho ecológico fundamental de esta especie

El análisis determinó que hay 4 localidades susceptibles a la reforestación (La Joya, Laguna Verde, Cerro Mozo y El Temascal); sin embargo, fueron detectadas diversas áreas donde los bosques que han sido objeto de degradación forestal y deforestación presentan aptitud para realizar labores de reforestación con fines de restauración o para el establecimiento de plantaciones forestales.

Por sus condiciones, en 2 localidades (Nueva Victoria y Agua Zarca) se pueden llevar a cabo acciones de conservación, puesto que sólo requieren manejo forestal orientado al aprovechamiento sustentable. Cabe señalar que existen rodales de aprovechamiento en los que pueden introducirse 2 individuos de esta especie para atenuar la erosión del suelo. Finalmente, el análisis realizado en el perfil bioclimático de las poblaciones de $P$. martinezii y el modelo de distribución potencial, determinó que existen 3 sitios óptimos para la recolección de semillas y establecimiento de viveros (Nueva Victoria, Agua Zarca y Laguna Verde).

A diferencia de varios trabajos previos, en éste se modeló la distribución de una especie de pino con datos de mayor resolución espacial y se validó el resultado utilizando un símil del valor de importancia relativa. El nivel de confianza del escenario 3 fue de $81.25 \%$, considerado aceptable.

$\mathrm{Si}$ bien el modelo requiere del reconocimiento y evaluación de las relaciones observadas entre factores biofísicos, éste se basó en una técnica de análisis multicriterio, más que multivariante. El análisis multicriterio espacialmente distribuido, y la resolución de los datos permitieron identificar unidades espaciales más 
continuas y que no se refieren a píxeles, relativamente aislados. Cabe resaltar que el escenario más confiable fue aquel que se construyó utilizando el proceso de análisis jerárquico, el cual permite identificar la consistencia de las comparaciones realizadas.

La importancia relativa (pesos) en todos los modelos siempre identificó que el grupo de criterios geopedológicos son más importantes para alcanzar la meta (distribución potencial). Los valores más altos correspondieron a andosoles, ignimbritas y complejos de laderas de lomeríos altos, seguidos por el grupo de criterios morfométricos, donde los valores más altos se relacionan con la altitud, la pendiente y la exposición de laderas; finalmente, el grupo menos relevante correspondió al climático, que incluyo la temperatura media y la precipitación.

Lo anterior sugiere, que la variabilidad climática no es tan alta en la zona estudiada; por el contrario, la diversidad geopedológica, seguida por la morfométrica, diferencian con mayor importancia la distribución de $P$. martinezii.

Los resultados de este estudio proporcionan información valiosa para la ejecución de posteriores estrategias de restauración que incluyan actividades de conservación y reforestación; tomando en cuenta diversos factores ambientales que determinan la aptitud del territorio para la especie. Los resultados también pueden apoyar la programación de futuras exploraciones en busca de nuevas poblaciones de P. martinezii que no han sido registradas, así como la introducción de esta especie en sitios que presentan condiciones ambientales idóneas.

Finalmente, cabe mencionar que el enfoque utilizado en este trabajo es reproducible a escalas menores; por ejemplo, de provincias fisiográficas $(1: 1000000)$ o país (1:4 000000$)$, considerando la disponibilidad y detalle de los datos espaciales y climáticos existentes, no sólo en México, sino en un buen número de países en vías de desarrollo.

\section{Agradecimientos}

Los autores de esta investigación agradecen al programa DGAPA-PAPPIT de la UNAM, clave IN304408. Esta investigación también fue parcialmente apoyada por CONACYT, clave 2005-C01-011. El primer autor agradece al CONACYT por la beca otorgada durante la realización de su maestría en Geografía, así como al Biól. Antonio Moreno Talamantes por el apoyo proporcionado durante la modelación espacial. Agradecemos los valiosos comentarios de los revisores anónimos y del Dr. Alfonso Delgado Salinas que mejoraron sustancialmente la versión original del manuscrito.

\section{Literatura citada}

Al- Zu'bi, Y. A. 2007. Application of multicriteria analysis for ranking and evaluation of waste water treatment plants and its impact on the environment and public health: Case study from Jordan. Journal of Applied Sciences Research 3:155-160.

Alba-Sánchez, F., J. A. López-Sáez, B. Benito de Pando, J. C. Linares, D. Nieto-Lugilde y L. López-Merino. 2010. Past and present potential distribution of the Iberian Abies species: a phytogeographic approach using fossil pollen data and species distribution models. Diversity and Distributions 16:214-228.

Anderson, R. P., A. T. Peterson y M. Gómez-Laverde. 2002. Using niche-based GIS modeling to test geographic predictions of competitive exclusion and competitive release in South American pocket mice. Oikos 98:3-6.

Aráujo, M. B. y A. Guisan. 2006. Five (or so) challenges for species distribution modelling. Journal of Biogeography 33:1677-1688.

Bonham-Carter, G. F. 1994. Geographic information systems for geoscientists: modelling with GIS. Geological Survey of Canada. Pergamon, Toronto. 398 p.

Brito, J. C., A. L. Acosta, F. Álvares y F. Cuzin. 2009. Biogeography and conservation of taxa from remote regions: an application of ecological-niche based models and GIS to North-African Canids. Biological Conservation 142:3020-3029.

Carranza, E. 1987. Aspectos botánico-ecológicos del campo geotérmico Los Azufres, Michoacán, México. Tesis, Escuela de Biología, Universidad Michoacana de San Nicolás de Hidalgo, Morelia, Michoacán. 60 p.

Chapman, S. B. 1976. Methods in plant ecology. Blackwell Scientific, Osney Mead, Oxford. 580 p.

Chefaoui, R. M., J. Hortal y J. M. Lobo. 2005. Potential distribution modelling, niche characterization and conservation status of Iberian Copris species in central Spain. Biological Conservation 122:327-338.

Chefaoui, R.M. y J. M. Lobo. 2008. Assessing the effects of pseudoabsences on predictive distribution model performance. Ecological Modelling 210:478- 486.

Cox, B. y D. Moore. 1994. Biogeography, an ecological and evolutionary approach. Blackwell Scientific, Oxford. 428 p.

Curtis, J. T. 1959. The vegetation of Wisconsin. An ordination of plant communities. University of Wisconsin Press, Madison. $657 \mathrm{p}$.

Farjon, A., J. A. Pérez de la Rosa y B. T. Styles. 1997. A field guide to the pines of Mexico and Central America. The Royal Botanic Gardens, Kew. 147 p.

Farjon, A. y B. T. Styles. 1997. Pinus (Pinaceae). Flora Neotropica. Monograph 75. The New York Botanical Garden, New York. 239 p.

Finch, J. M., M. J. Samways, T. R. Hill, S. E. Piper y S. Taylor. 2005. Application of predictive distribution modeling to invertebrates: Odonata in South Africa. Biodiversity and 
Conservation 15:4239-4251.

Fischer, J., D. B. Lindenmayer, H. A. Nix, J. L. Stein y J. A. Stein. 2001. Climate and animal distribution: a climatic analysis of the Australian marsupial Trichosurus caninus. Journal of Biogeography 28:293-304.

García, E. 2004. Modificaciones al sistema de clasificación climática de Köppen. Instituto de Geografía, UNAM, México, D. F. 91 p.

Garduño-Monroy, V. H. e I. Isra-de Alcántara. 2010. Características biofísicas de la cuenca del lago de Cuitzeo: Geología. In Atlas de la cuenca del lago de Cuitzeo: un análisis de la geografía del lago y su entorno socioambiental, S. Cram, L. Galicia e I. Isra-de Alcántara, (comps). Universidad Nacional Autónoma de México, México, D. F. p. 28-33.

Geneletti, D. 2004. A GIS-based decision support system to identify nature conservation priorities in an alpine valley. Land Use Policy 21:149-160.

Geneletti, D. 2005. Formalising expert's opinion through multiattribute value functions. An application in landscape ecology. Journal of Environmental Management 76:255-262.

Geneletti, D. 2007. An approach based on spatial multicriteria analysis to map the nature conservation value of agricultural land. Journal of Environmental Management 83:228-235.

Geneletti, D. 2009. Combining stakeholder analysis and spatial multicriteria evaluation to select and rank inert landfill sites. Waste Management 30:328-337.

ESRI. 1999. ArcView 3.2. GIS. Environmental Systems Research Institute, New York.

ESRI. 2008. ArcGis 9.2. User's guide. Environmental Systems Research Institute, Redlands, California.

Graham, C. H., S. Ron, J. C. Santos y C. J. Schneider. 2004. Integrating phylogenetics and environmental niche models to explore speciation mechanism in dendrobatid frogs. Evolution 58:781-1793.

Hanson, H. C. y E. Churchill D. 1961. The plant community. Reinhold, New York. 218 p.

Hutchinson, G. E. 1957. Concluding remarks. Cold Spring Harbor Symposia Quantitative Biology 22:415-427.

Illoldi-Rangel, P., M. A. Linaje y V. Sánchez-Cordero. 2002. Distribución de los mamíferos terrestres en la región del golfo de California, México. Anales del Instituto de Biología, Universidad Nacional Autónoma de México. Serie Zoología 73:213-224.

Illoldi-Rangel, P., V. Sánchez-Cordero y A. T. Peterson. 2004. Predicting distribution of Mexican mammals using ecological niche modeling. Journal of Mammalogy 85:658-662.

INEGI. 1999. Conjunto de datos vectoriales. Instituto Nacional de Estadística Geografía e Informática, Aguascalientes, Aguascalientes.

INEGI. 2004. Guía para la interpretación de la cartografía. Edafología. Instituto Nacional de Estadística Geografía e Informática, Aguascalientes, Aguascalientes. 28 p.
ITC. 2005. ILWIS 3.3 Integrated land and water information system, GIS software. International Institute for GeoInformation Science and Earth Observation, Enschede. [The Netherlands]. 520 p.

Jennings, M. D. 2000. Gap analysis: concepts, methods and recent results. Landscape Ecology 15:5-20.

Kearney, M. y W. P. Porter. 2004. Mapping the fundamental niche: physiology, climate, and the distribution of a nocturnal lizard. Ecology:3119-3131.

Keeney, R. L. 1992. Value-focused thinking, a pad to creative decision making. Harvard University Press, Cambridge, Massachusetts. 416 p.

Larsen E. 1964. A new species of pine from Mexico. Madroño 17:217-8.

Leal-Nares, O. A., M. E. Mendoza y E. Carranza González. 2010. Análisis y modelamiento espacial de información climática en la cuenca de Cuitzeo, México. Investigaciones Geográficas 72:49-67.

López-Granados, E., G. Bocco, M. E. Mendoza, A. Velázquez y R. Aguirre. 2006. Peasant emigration and land-use change at the watershed level. A GIS-based approach in Central Mexico. Agricultural Systems 90:62-78.

Madrigal-Sánchez, X. 1994. Características ecológicas forestales de la región forestal oriental del estado de Michoacán; México. Universidad Michoacana de San Nicolás de Hidalgo, Morelia, Michoacán. 114 p.

Madrigal-Sánchez, X. y L. I. Guridi-Gómez. 2004. Riqueza de la vegetación en la región de Morelia. In Contribuciones a la geología e impacto ambiental en la región de Morelia, V. H. Garduño (ed.). Instituto de Investigaciones Metalúrgicas, Universidad Michoacana de San Nicolás de Hidalgo, Morelia, Michoacán. p. 156-166.

Malczewski, J. 1997. Propagation of errors in multicriteria location analysis: a case study. In Multiple criteria decision making. Proceedings of the Twelfth International Conference. Hagen (Germany). 1995, G. Fandel y T. Gal (eds.). Springer, Berlin. p. 154-165.

Malczewski, J. 1999. GIS and multicriteria decision analysis. Wiley, New York. 392 p.

Matteucci, S. y A. Colma. 1982. Metodología para el estudio de la vegetación. Secretaría General de los Estados AmericanosPrograma Regional de Desarrollo Científico y Tecnológico, Washington, D.C. 168 p.

Mazerolle, M. J. y M. A. Villard. 1999. Patch characteristics and landscape context as predictors of species presence and abundance: a review. Ecoscience 61:117-124.

Mendoza, G. A. y P. Macoun. 1999. Guidelines for applying multicriteria analysis to the assessment of criteria and indicators. Center for International Forestry Research, Bogor. 82 p.

Mendoza, M. E., E. López-Granados y G. Bocco. 2001. Regionalización ecológica, conservación de recursos y ordenamiento territorial. Informe final presentado al Programa 
SIMORELOS- CONACYT. Departamento de Ecología de los Recursos Naturales, Instituto de Ecología, UNAM, México, D. F. 266 p.

Mendoza, M. E. 2002. Implicaciones del cambio de cobertura vegetal y uso del suelo en el balance hídrico a nivel regional. El caso de la cuenca del lago de Cuitzeo. Tesis, Doctorado Instituto de Geofísica, UNAM, México, D. F. 188 p.

Mendoza, M. E., G. Bocco, M. Bravo, E. López-Granados y W. R. Osterkamp. 2006. Predicting water surface fluctuation of continental lakes. A GIS and RS based approach in Central Mexico. Water Resources Management 20:291-311.

Mendoza, M. E., D. I. González-Terrazas, D. Geneletti, L. M. Morales-Manilla, E. López, I. Israde y Z. Vekerdy. 2008. Uso de técnicas de análisis multicriterio para la priorización de subcuencas y municipios para la conservación, restauración y el aprovechamiento de los recursos naturales en la cuenca del lago de Cuitzeo (Clave 2005-01-011). Informe final presentado al Fondo Mixto CONACYT-Estado de Michoacán. Centro de Investigaciones en Geografía Ambiental, UNAM, México, D. F. 110 p.

Mendoza, M. E., G. Bocco, E. López y M. Bravo. 2010. Hydrological implications of land-cover and land-use change: Spatial analytical approach at regional scale in the closed basin of the Cuitzeo Lake, Michoacan, Mexico. Singapore Tropical Geography 31:197-214.

Mendoza, M. E. y G. Bocco. 2010. Características biofísicas de la cuenca del lago de Cuitzeo: geomorfología. In Atlas de la cuenca del lago de Cuitzeo: un análisis de la geografía del lago y su entorno socioambiental, S. Cram, L. Galicia e I. IsradeAlcántara (comps.). Universidad Nacional Autónoma de México, México, D. F. p. 48-53.

Mitov, P. G. e I. L. Stoyanov. 2005. Ecological profiles of harvestmen (Arachnida, Opiliones) from Vitosha Mountain (Bulgaria): a mixed modelling approach using gams. The Journal of Arachnology 33:256-268.

Paegelow, M., M. T. Camacho-Olmedo y J. Menor-Toribio. 2003. Cadenas de Markov, evalución multicriterio y evaluación multiobjetivo para la modelización prospectiva del paisaje. GeoFocus 3:22-44.

Petraglia, A. y M. Tomaselli. 2003. Ecological profiles of wetland plant species in the northern Apennines (N. Italy). Journal of Limnology 62:71-78.

Phillips, S. y M. Dudík. 2008 Modeling of species distributions with Maxent: new extensions and a comprehensive evaluation. Ecography 31:161-175.

Ricker, M. y H. M. Hernández. 2010. Tree and tree-like species of Mexico: gymnosperms, monocotyledons, and tree ferns. Revista Mexicana de Biodiversidad 81:27-38.

Saaty, T. L. 1980. The analytic hierarchy process. McGraw-Hill, New York. 165 p.

Scoble, J. y A. J. Lowe. 2010. A case for incorporating phylogeography and landscape genetics into species distribution modelling approaches to improve climate adaptation and conservation planning. Diversity and Distributions 16:343-353.

SEMARNAT (Secretaría de Medio Ambiente y Recursos Naturales). 2010. Norma oficial mexicana. NOM-059. Protección ambiental - Especies nativas de México de flora y fauna silvestres -Categorías de riesgo y especificaciones para su inclusión, exclusión o cambio - Lista de especies en riesgo. Diario Oficial de la Federación, 30 de diciembre: 1-78.

Sharifi, A. y M. Herwijnen, 2004. Spatial decisión support systems. ITC Lectures. ITC, Enschede. 234 p.

Silva, M. A. 2005. Distribución y aspectos ecológicos de Pinus martinezii Larsen, en el Eje Neovolcánico, Estado de Michoacán, México. Tesis, Facultad de Biología, Universidad Michoacana de San Nicolás de Hidalgo, Morelia, Michoacán. $88 \mathrm{p}$.

Soberon, J. y M. Nakamura. 2009. Niches and distributional areas: concepts, methods, and assumptions. Proceedings of the National Academy of Science of the United States of America 103:19644-19650.

Stockman, A.K., D.A. Beamer y J.E. Bond. 2006. An evaluation of a GARP model as an approach to predicting the spatial distribution of non- vagile invertebrate species. Diversity and Distributions 12: 81-89.

Stockwell, B. R. y P. A. Townsend. 2002. Effects of sample size on accuracy of species distribution Models Ecological Modelling 148:1-13.

Styles, B. T. 1998. El género Pinus: su panorama en México. In Diversidad biológica de México, T. P. Ramamoorthy, R. Bye, A. Lot y J. Fa (eds.). Instituto de Biología, UNAM, México, D. F. p. 385-408.

Townsend, P. A. 2001. Predicting species' geographic distributions based on ecological niche modeling. The Condor 103:599605.

Townsend, P. y D. A. Klusa. 2003. New distributional modelling approaches for gap analysis. Animal Conservation. The Zoological Society of London 6:47-54.

Vaughton, G. y M. Ramsey. 2004. Dry environments promote the establishment of females in monomorphic populations of Wurmbea biglandulosa (Colchicaceae). Evolutionary Ecology 18:323-341.

Villaseñor, J. L. y O. Téllez-Valdéz. 2004. Distribución potencial del género Jefea (Asteraceae) en México. Anales del Instituto de Biología, Universidad Nacional Autónoma de México, Serie Botánica 75:205-220.

Whittaker H. R. 1975. Communities and ecosystems. Macmillan, New York. 387 p.

Williams, P. H., C. R. Margules y D. W. Hilbert. 2002. Data requirements and data sources for biodiversity priority area selection. Journal of Bioscience 27:327-338. 